\title{
Segment-orientated analysis of two-dimensional strain and strain rate as assessed by velocity vector imaging in patients with acute myocardial in- farction
}

\author{
Thomas Butz ${ }^{\star}$, Corinna N. Lang*, Marc van Bracht, Magnus W. Prull, Hakan Yeni, Petra Maagh, Gunnar \\ Plehn, Axel Meissner, Hans-Joachim Trappe
}

Department of Cardiology and Angiology, Marienhospital Herne, Ruhr University Bochum, Hoelkeskampring 40, D-44625

Herne, Germany

* Both authors contributed equally to this work

Corresponding author: Thomas Butz, MD, Department of Cardiology and Angiology, Marienhospital Herne, Ruhr-University Bochum, Hoelkeskampring 40, D-44625 Herne, Germany. Phone: +49 (0)2323 499-0; Fax: +49 (0)2323 499-360; Mail: Thomas.Butz@Marienhospital-Herne.de

Received: 2010.11.14; Accepted: 2011.01.31; Published: 2011.02.01

\begin{abstract}
Aims: Strain rate imaging techniques have been proposed for the detection of ischemic or viable myocardium in coronary artery disease, which is still a challenge in clinical cardiology. This retrospective comparative study analyzed regional left ventricular function and scaring with two-dimensional strain (2DS) in the first 4 to 10 days after acute anterior myocardial infarction (AMI).

Methods and results: The study population consisted of 32 AMI patients with an LAD occlusion and successful reperfusion. The assessment of peak systolic 2DS and peak systolic strain rate (SR) was performed segment-oriented with the angle-independent speckle tracking algorithm Velocity Vector Imaging (VVI). The infarcted, adjacent and non-infarcted segments were revealed by late enhancement MRI (LE-MRI), which was used as reference for the comparison with 2DS. The infarcted segments showed a significant decrease of tissue velocities, 2DS and SR in comparison to the non-affected segments.

Conclusion: 2DS and SR as assessed by VVI seem to be a suitable approach for echocardiographic quantification of global and regional myocardial function as well as a promising tool for multimodal risk stratification after anterior AMI.
\end{abstract}

Key words: Myocardial infarction, Two-dimensional strain, Strain rate imaging, Late Enhancement MRI

\section{Introduction}

After acute myocardial infarction (AMI) the discrimination of avital scar tissue and vital reversible harmed myocardium is crucial for the optimal individual therapy, and for risk stratification ${ }^{1}$. Further intervention, such as a percutaneous coronary intervention (PCI) or a coronary artery bypass graft (CABG), is only indicated if the myocardium is hypokinetic due to insufficient blood supply ("hiber- nating" or "stunned" myocardium), but still viable. Until now only late enhancement magnetic resonance imaging (LE-MRI) has provided a certain distinction. However, its application is still limited due to high expense and restricted availability. Therefore, current studies are mostly concerned with the question if newly emerged parametric echocardiographic methods, measuring left ventricular (LV) function and vi- 
ability by the deformation indices two-dimensional strain (2DS) and strain rate (SR), might be an alternative approach in clinical routine.

Tissue Doppler Imaging (TDI) and 2D Speckle Tracking (2DST) algorithms facilitate the assessment of tissue velocities and deformation markers. But only strain - procentual length alteration relative to a base length - can distinguish between active and passive movement. Strain rate records the change of length per time unit ${ }^{2-6}$.

Previous studies using TDI or 2DST demonstrated a reduction of the myocardial deformation indices 2DS and SR in infarcted segments after AMI 7-13. The goal of our retrospective study - since only limited data has been obtained thus far - was the further validation of the 2DST software "Velocity Vector Imaging" (VVI) for the differentiation between infarcted and non-infarcted segments as well as, the correct localization of the infarcted segments in comparison to LE-MRI 14-15.

\section{Methods}

Patients with their first AMI and successful reperfusion of the left anterior descending artery (LAD) by primary PCI were retrospectively enrolled in this study.

Inclusion criteria were AMI caused by LAD occlusion (type I, ESC) and coronary artery disease affecting only 1 or 2 vessels. Patients with previous AMI, with 3 affected coronary arteries, after CABG, non-ischemic cardiomyopathy or high grade valvular disease were excluded.

Standard echocardiography and cardiac LE-MRI were performed 4 to 10 days after AMI. The segments were categorized by cardiac LE-MRI as follows: infarcted (LE $51-100 \%$ of wall thickness and LAD territory), adjacent (either LE 1-50\% of wall thickness or no LE but LAD perfusion territory) and non-infarcted
(LE $0 \%$, no LAD perfusion territory $)^{9}$. The results of the VVI offline analysis of the tissue velocities $\left(\mathrm{S}^{\prime}, \mathrm{E}^{\prime}\right.$, $\left.\mathrm{A}^{\prime}\right)$ derived from 2DST and deformation markers (2DS, 2DSR) were compared intra-individually to the MRI findings.

The study protocol was approved by the local ethics committee of the Ruhr-University of Bochum.

\section{Conventional 2D Doppler Echocardiography}

In left lateral decubital position the patients underwent transthoracic echocardiography according to the ASE guidelines ${ }^{16}$ on a Sequoia C512 ultrasound system (Siemens Healthcare, Erlangen, Germany) equipped with a phased array transducer (frequency range of $3.75-4.25 \mathrm{MHz}$ ). ECG-controlled parasternal long axis, parasternal short axis, apical 4-, 3-, and 2-chamber views of LV walls were obtained in endexspiration. 3 cardiac cycles of each view were digitally stored with the KardioPACS-Software 7.0 (medPACS, Essen, Germany). LV ejection fraction (LV-EF) was calculated by the modified Simpson's method. LA and LV diameter were measured by M-Mode echocardiography. High grade cardiac valvular disease was excluded by Color, PW and CW Doppler according to current guidelines ${ }^{16}$.

\section{Velocity vector imaging (VVI)}

The principle of angle-independent VVI (Siemens, Erlangen, Germany) has been developed from M-Mode modifications ${ }^{17}$. Using 2D gray scale images VVI analysis can be carried out in order to measure tissue velocities ( $\left.\mathrm{S}^{\prime}, \mathrm{E}^{\prime}, \mathrm{A}^{\prime}\right), 2 \mathrm{DS}$ and SR. We employed the ASE recommendation of a 17 segments model for our research to examine MRI and Echo data ${ }^{18}$. The observer defined the endocardial border manually and placed regions of interests (ROI) in the middle of every segment (see Figure 1).

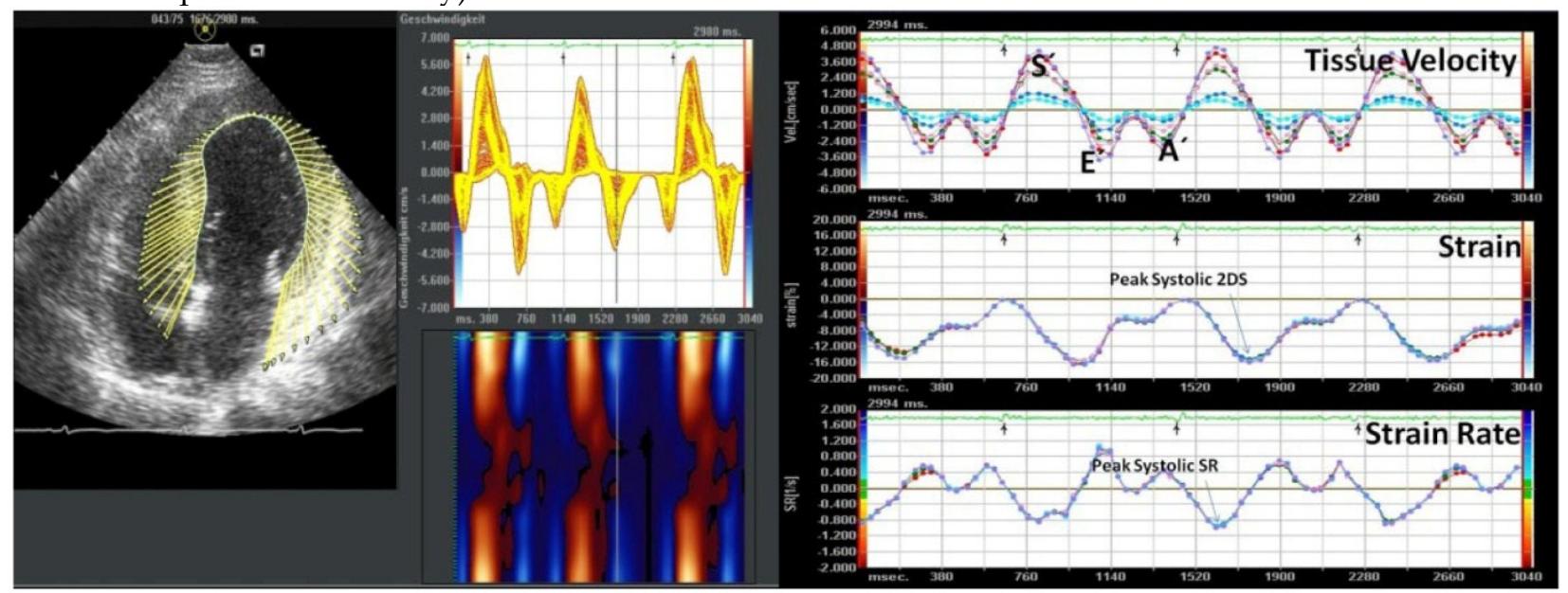

Figure I: VVI approach to tissue velocities and deformation in the left ventricle (Four chamber view) 
The endocardial border and the myocardium was then automatically tracked frame-by-frame by the VVI software throughout the cardiac cycle. The VVI algorithm includes speckle tracking, global motion coherence, and consistency of periodicity between cardiac cycles, which are described in detail in the producers patent (US 6.909.914) and the patent application publications (US 2005/0070798, US 2005/0074153) ${ }^{19-20 .}$

In our study we focused on the longitudinal velocities and deformation markers because ischemia especially affects subendocardial fibers first, which are mainly responsible for longitudinal movement 21 .

\section{Late enhancement magnetic resonance imaging (LE-MRI)}

Using a 1.5-Tesla Magnetom Sonata system (Siemens, Erlangen, Germany) we scanned the heart and surrounding structures of 32 patients ECG-triggered in endexspiration and produced the standard views of the long and short axes, as well as the left ventricular outflow tract. With the CMRtools software (Cardiovascular Imaging Solutions, London, UK) we calculated volumes as well as the LV-EF of the left ventricle according to the reference data of Maceira et al. ${ }^{22}$.

Late gadolinium enhancement images were acquired 10 to 25 minutes after applicating 0.1-0.2 $\mathrm{mmol} / \mathrm{kg}$ bodyweight Magnevist ${ }^{\circledR}$ (Bayer, Leverkusen, Germany) with a 2D-segmented, spoilt, turbo gradient echo sequence (TRUFISP, Siemens, Erlangen, Germany). This sequence technique developed by Simonetti et al. allows a detection of myocardial necrosis, scars and fiber tissue (hyperenhancement of the myocardium) ${ }^{30}$. The inversion time was individually adapted.

The segments were labeled in infarcted (LE $51-100 \%$ of wall thickness), adjacent (either LE 1-50\% or no LE but LAD perfusion territory) and non-infarcted (LE $0 \%$, no LAD perfusion territory) as proposed before ${ }^{9}$.

\section{Statistics}

All continuous values were expressed as mean \pm standard deviation after ascertaining a normal distribution. We performed the unpaired or paired $t$-tests and one-way repeated measures analysis of variance (ANOVA). If the ANOVA test results were significant we followed up with the post hoc Scheffé procedure. ROC analysis was performed as previously described 23. Coefficients of variance were calculated for the inter- and intra-observer variation. Differences were considered significant when the p-value was less than
0.05. We used the statistic software SPSS 15.0 (Chicago, IL, USA) for all analyses.

\section{Results}

Between August 2006 and April 2009, 32 patients (27 men) with a mean age of 58 \pm 12 years (range 38-81 years), who had their first anterior AMI (23 STEMI; 9 NSTEMI) and underwent successful reperfusion of the LAD by PCI, were enrolled in this study. Successful acute revascularization of the infarcted area was achieved by recanalisation, PCI and Stenting of the culprit lesion in the infarct-related artery (LAD). No patient had to underwent CABG. In the 11 patients with 2 vessel disease a stenosis $>50 \%$ was found in the circumflex artery in 4 patients and in the right coronary artery in 7 patients. Complete revascularization in the patients with 2-vessel disease was achieved by serial PCI of the remaining diseased coronary arteries according to hemodynamic relevance and morphology of the stenosis during the further clinical course. Basic clinical data are listed in Table 1.

Table I: Basic clinical characteristics

\begin{tabular}{ll}
\hline Gender (male/female) & $27 / 5$ \\
Age (years) & $58 \pm 12$ \\
\hline Height $(\mathrm{cm})$ & $1.72 \pm 9$ \\
Weight $(\mathrm{kg})$ & $81 \pm 15$ \\
BMI $\left(\mathrm{kg} / \mathrm{m}^{2}\right)$ & $27 \pm 4$ \\
ECG $(\mathrm{STEMI} / \mathrm{NSTEMI})$ & $23 / 9$ \\
\hline
\end{tabular}

Echocardiography was performed $7.8 \pm 3.6$ days after AMI. With a mean of $49 \pm 12 \%$, the LV-EF was mildly impaired after the AMI. M-Mode and B-Mode transthoracic echocardiographic diameters and volumes are displayed in Table 2.

Table 2: Echocardiographic data set

\begin{tabular}{ll}
\hline Ejection fraction, EF $(\%)$ & $49 \pm 12$ \\
IVSD $(\mathrm{cm})$ & $1.1 \pm 0.2$ \\
HWD $(\mathrm{cm})$ & $1.0 \pm 0.1$ \\
LVDD $(\mathrm{cm})$ & $5.2 \pm 0.4$ \\
LVSD $(\mathrm{cm})$ & $3.7 \pm 1.2$ \\
LV-EDV $(\mathrm{ml})$ & $166 \pm 46$ \\
FS $(\%)$ & $29 \pm 12$ \\
LA $(\mathrm{cm})$ & $3.8 \pm 0.6$ \\
RVDD $(\mathrm{cm})$ & $2.1 \pm 0.7$ \\
Aorta $(\mathrm{cm})$ & $3.0 \pm 0.5$ \\
\hline
\end{tabular}

\section{Vector Velocity Imaging (VVI)}

In $386(71 \%)$ of 544 segments the analysis by VVI was feasible. Segments were excluded if the endocar- 
dial border was not tracked properly, if the digital storage of 3 cardiac cycles was not completed, and if movement of the files was evoked by breathing excursions of the patient. The mean picture frame rate (PFR) was $45 \pm 16 \mathrm{~s}^{-1}$. The average values for the global longitudinal deformation were: 2DS $-11.67 \pm 5.38 \%$; systolic SR (sSR) $-0.65 \pm 0.27 \mathrm{~s}^{-1}$; early diastolic SR (Sre) $0.60 \pm 0.35 \mathrm{~s}^{-1}$.

The analysis of tissue velocities demonstrated a gradient of systolic (S') and diastolic (early $\mathrm{E}^{\prime}$ and late $\mathrm{A}^{\prime}$ ) velocities from the apex to the basis of the heart with significant differences between basal, midventricular and apical myocardium (see Table 3).

Table 3: Tissue velocities $\left(S^{\prime}, E^{\prime}, A^{\prime}\right)$ of basal, mid and apical segments as assessed by VVI

\begin{tabular}{lllll}
\hline & Basal & Mid & Apical & p ANOVA \\
$\mathrm{S}^{\prime}(\mathrm{cm} / \mathrm{s})$ & $3.64 \pm 1.63$ & $2.41 \pm 1.07$ & $1.06 \pm 0.65$ & $\mathrm{p}<0.001$ \\
$\mathrm{E}^{\prime}(\mathrm{cm} / \mathrm{s})$ & $-2.60 \pm 1.37$ & $-1.68 \pm 0.91$ & $-0.72 \pm 0.67$ & $\mathrm{p}<0.001$ \\
\hline
\end{tabular}

\section{Comparison of VVI and LE-MRI}

MRI was performed $8.1 \pm 1.4$ days after AMI. The LE-MRI study resulted in 209 (38\%) segments with a LE $\geq 51 \%, 91(17 \%)$ with a LE of $1-50 \%$ and $244(45 \%)$ segments without LE. The categorization labeled 209 segments (38\%) as infarcted, $162(30 \%)$ as adjacent and $173(32 \%)$ as non-infarcted.

The comparison of infarcted and non-infarcted segments showed a significant difference $(p<0.05)$ according to 2DS, dSR and tissue velocities, which is depicted in Table 4 and Figures 2, 3. Infarcted segments demonstrated significantly decreased 2DS as well as tissue velocities in comparison to adjacent and non-infarcted segments.

To investigate infarct transmurality we also compared the segments with LE $\geq 51 \%$, LE $1-50 \%$ and no LE. We demonstrated significant differences of 2DS, dSR, $S^{\prime}, E^{\prime}, A^{\prime}(p<0.05)$ between segments with $\mathrm{LE} \geq 51 \%$ and segments with no LE. According to tissue velocity values we additionally found significant differences between segments with LE $\geq 51 \%$ and LE $1-50 \%(p<0.05)$. The data is presented in Table 5.

In a receiver operating characteristic curve (ROC) analysis VVI-derived mean peak systolic velocity $S^{\prime}$ of all infarcted segments in comparison to the mean peak $S^{\prime}$ velocities of the adjacent and non-infarcted segments predicted infarction (LE $\geq$ $51 \%$ ) with $80 \%$ sensitivity and $70 \%$ specificity (area under the curve, AUC: 0.8, confidence interval $0.77-0.86$ ) for a cut-off value less than $1.95 \mathrm{~cm} / \mathrm{s}$ (Figure 4, Table 6).
A:

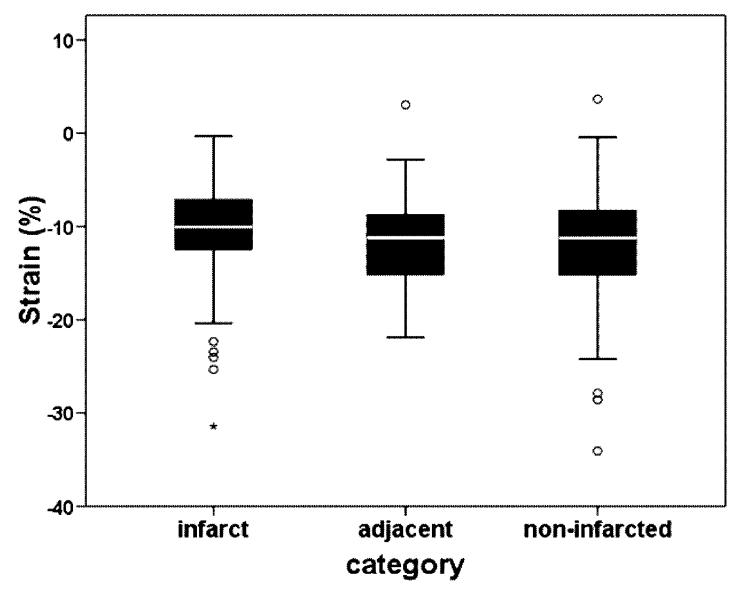

B:

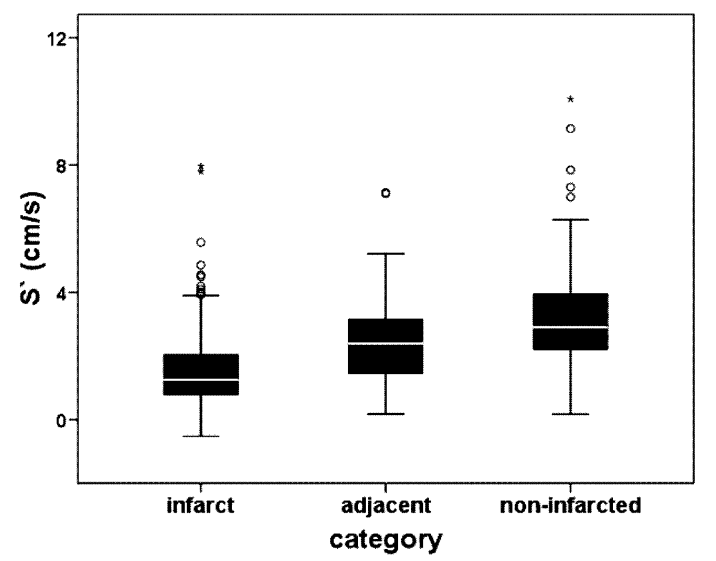

Figure 2: Significant difference of Strain (A; ANOVA: $p$ < 0.05 ) and $S^{\prime}$ (right) between infarcted, adjacent and non-infarcted segments (B; ANOVA: $p<0.01$ )

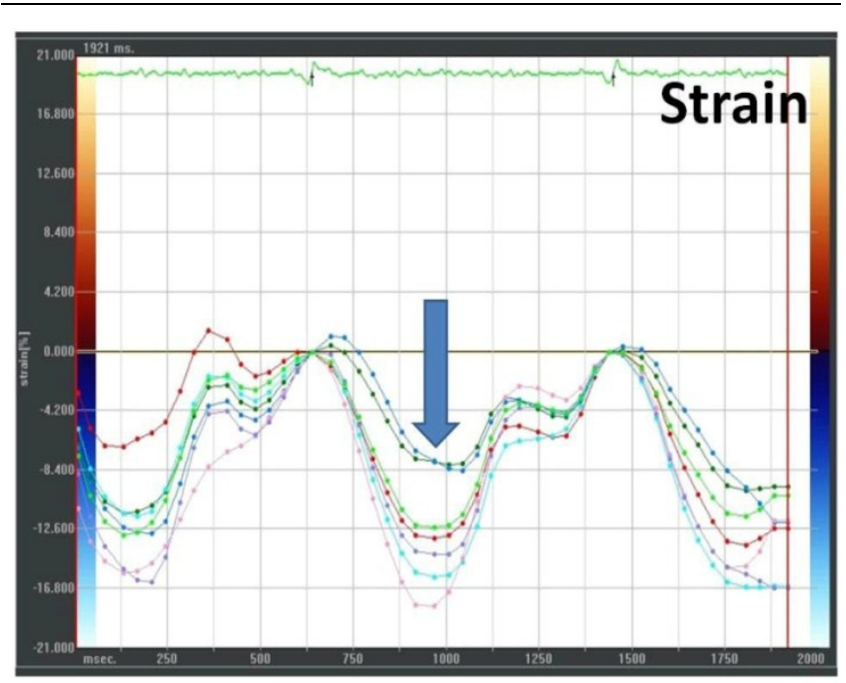

Figure 3: Example of a VVI analysis with markedly reduced strain (arrow) in septal segments after AMI (four-chamber view; green and blue $\mathrm{ROI}$ representing the mid and apical septal segments) 


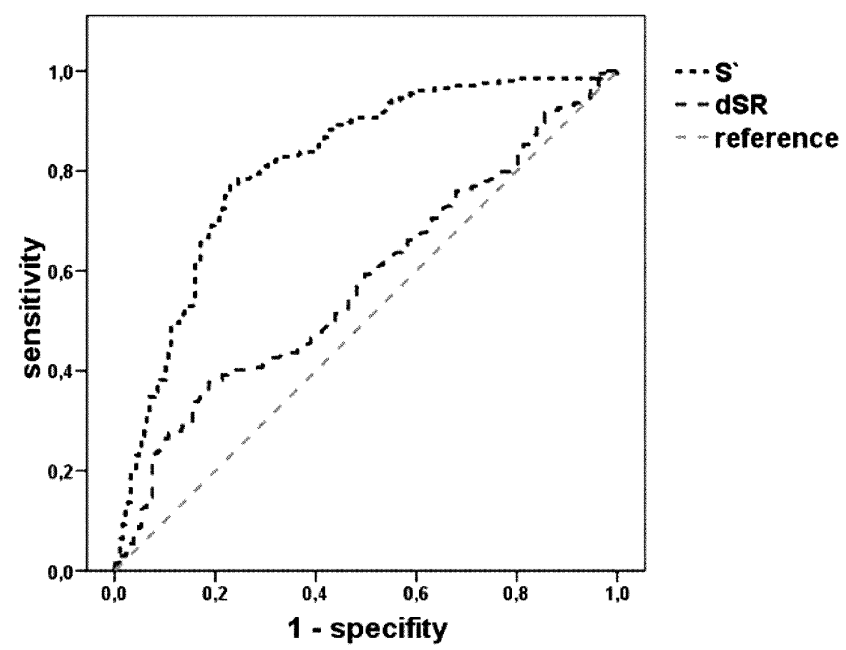

Table 5: Comparison of deformation imaging and infarct transmurality by LE-MRI

\begin{tabular}{|c|c|c|c|c|}
\hline & No LE & LE $1-50 \%$ & $\mathrm{LE} \geq 51 \%$ & p ANOVA \\
\hline 2DS (\%) & $-11.87 \pm 5.42$ & $-11.73 \pm 4.28$ & $-10.34 \pm 4.76$ & $p<0.05$ \\
\hline $\operatorname{sSR}\left(\mathrm{s}^{-1}\right)$ & $-0.66 \pm 0.26$ & $-0.69 \pm 0.26$ & $-0.62 \pm 0.25$ & n.s. \\
\hline $\operatorname{SRe}\left(\mathrm{s}^{-1}\right)$ & $0.69 \pm 0.26$ & $0.58 \pm 0.34$ & $0.53 \pm 0.32$ & $p<0.05$ \\
\hline$S^{\prime}(\mathrm{cm} / \mathrm{s})$ & $3.01 \pm 1.64$ & $2.30 \pm 1.25$ & $1.60 \pm 1.26$ & $p<0.001$ \\
\hline$E^{\prime}(\mathrm{cm} / \mathrm{s})$ & $-2.22 \pm 1.40$ & $-1.60 \pm 1.03$ & $1.10 \pm 0.94$ & $p<0.001$ \\
\hline$A^{\prime}(\mathrm{cm} / \mathrm{s})$ & $1.85 \pm 1.17$ & $-1.30 \pm 0.93$ & $-0.99 \pm 0.84$ & $p<0.001$ \\
\hline
\end{tabular}

Table 6: Receiver operating characteristic (ROC) analysis for different modalities for the detection of infarcted segments

\begin{tabular}{|l|llll|}
\hline & cut-off & AUC & sensitivity & specificity \\
\hline Strain (\%) & -12.00 & 0.6 & $70 \%$ & $43 \%$ \\
& -10.34 & 0.6 & $54 \%$ & $59 \%$ \\
\hline & -6.50 & 0.6 & $23 \%$ & $87 \%$ \\
\hline sSR $\left.\mathbf{s}^{-1}\right)$ & -0.73 & 0.54 & $70 \%$ & $36 \%$ \\
\hline SRe (s $\left.\mathbf{s}^{-1}\right)$ & 0.34 & 0.6 & $80 \%$ & $20 \%$ \\
\hline $\mathbf{S}^{\prime}(\mathbf{c m} / \mathbf{s})$ & 1.95 & 0.8 & $80 \%$ & $70 \%$ \\
\hline
\end{tabular}

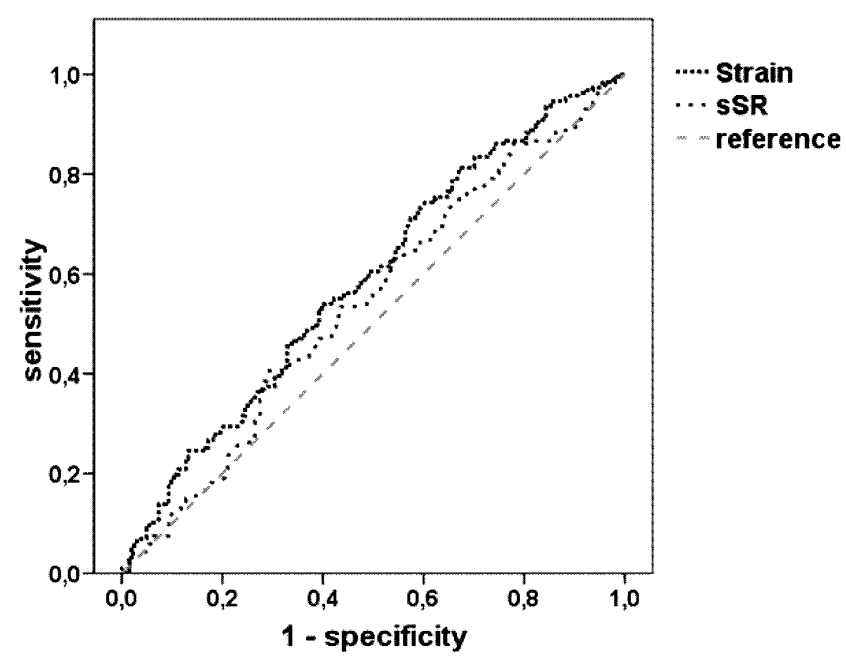

Figure 4: ROC analysis for the detection of previous segmental myocardial infarction by strain, sSR, dSR or $\mathrm{S}^{\prime}$ after AMI

Table 4: Comparison of deformation and tissue velocities in AMI according to the categorization of the segments as infarcted, adjacent and non-infarcted (see methods).

\begin{tabular}{lllll}
\hline & infarcted & adjacent & non-infarcted & p ANOVA \\
2DS (\%) & $-10.37 \pm 4.75$ & $-11.45 \pm 4.55$ & $-12.01 \pm 5.42$ & $\mathrm{p}<0.05$ \\
\hline $\mathbf{s S R}\left(\mathbf{s}^{-1}\right)$ & $-0.62 \pm 0.25$ & $-0.68 \pm 0.25$ & $-0.66 \pm 0.27$ & n.s. \\
SRe (s-1) & $0.53 \pm 0.32$ & $0.60 \pm 0.34$ & $0.63 \pm 0.40$ & $\mathrm{p}<0.05$ \\
$\mathbf{S}^{\prime}(\mathbf{c m} / \mathbf{s})$ & $1.61 \pm 1.27$ & $2.43 \pm 1.33$ & $3.10 \pm 1.66$ & $\mathrm{p}<0.001$ \\
$\mathbf{E}^{\prime}(\mathbf{c m} / \mathbf{s})$ & $-1.10 \pm 0.94$ & $-1.65 \pm 1.08$ & $-2.25 \pm 1.42$ & $\mathrm{p}<0.001$ \\
$\mathbf{A}^{\prime}(\mathbf{c m} / \mathbf{s})$ & $-1.00 \pm 0.84$ & $-1.36 \pm 0.96$ & $-1.88 \pm 1.19$ & $\mathrm{p}<0.001$ \\
\hline
\end{tabular}

For intra-observer variability the same observer reviewed the echocardiographic images of 20 patients and repeated VVI measurements several weeks after the initial measurement. In 8 cases we blinded a second observer to the first VVI measurements and MRI data for another review. The results were reported as correlation coefficients. For intra-observer variability we found a correlation coefficient of $11 \%$, for inter-observer variability we demonstrated a correlation coefficient of $17 \%$. A paired t-test did not confirm any significant difference between the obtained data sets.

\section{Discussion}

The main finding of this study is the significant difference of strain, SRe and tissue velocities between infarcted and non-infarcted segments demonstrated by an comparison between 2D Speckle Tracking (2DST) and late enhancement MRI (LE-MRI).

\section{Deformation imaging after AMI}

Our data demonstrated a significant difference of tissue velocities $\left(\mathrm{S}^{\prime}, \mathrm{E}^{\prime}\right)$ between infarcted and non-infarcted segments assessed by VVI. Mean peak systolic velocity $S^{\prime}$ predicted infarcted segments (LE $\geq$ $51 \%$ ) in comparison to adjacent and non-infarcted segments with a sensitivity of $80 \%$ and a specificity of $70 \%$ (AUC 0.8) with a cut-off value of less than 1.95 $\mathrm{cm} / \mathrm{s}$.

Poor agreement between VVI and TDI measurements have been previously demonstrated, and therefore they are not interchangeable 23,37. The measured tissue velocities in the present study are lower than in previous VVI studies ${ }^{14,15}$, which might 
be explained by the extent of infarct size or relatively lower PFR's in our study. At the moment there are no normal values of a large cohort of healthy controls and validated recommendations for the PFR in VVI analysis are still not available. The data of former studies showed that a mean PFR about $40-50 \mathrm{~Hz}$ is suitable for VVI analysis. A far higher PFR might not assure the proper capturing of the speckle pattern, and a lower frame rate might cause even decreased values. No relevant affection of the measurements due to the PFR should be expected because our analysis and interpretation of the results based on an intra-individual comparison of the segments.

As velocity measurements alone are not able to differentiate between active and passive movement, deformation imaging is crucial to identify ischemic tissue 7 , 24-29. In principle, our findings are confirming previously published studies about infarct detection and size, who described significant differences of the radial and circumferential strain and strain rate by 2DST in normo-, hypo-, akinetic and dyskinetic segments of post-infarct patients ${ }^{8-13,30-37}$, which were not reliably accessible by TDI. Radial strain also allowed the differentiation between transmural and non-transmural infarction ${ }^{31}$, a layer specification ${ }^{10}$, and the identification of segments, which will recover after a revascularization therapy 32 .

In addition, our data is supporting the findings of Chen et al. ${ }^{14}$, who were able to differentiate between segments of healthy controls, infarcted and non-infarcted segments of post AMI patients by VVI. Jurcut et al. ${ }^{15}$ also showed significant differences of infarcted and non-infarcted segments by VVI with LE-MRI as reference method. Additionally, they could differentiate between adjacent segments and infarcted segments as well as between adjacent and non-infarcted segments. Referring to the latter, we were able to show significant differences by tissue velocity analysis, but not by strain analysis between infarcted and adjacent segments.

Segments with LE $\geq 51 \%$ demonstrated significantly decreased strain and velocities in comparison to segments with no LE. Comparing segments with $\mathrm{LE} \geq 51 \%$ and LE $1-50 \%$ presented a significant difference of tissue velocities ( $\mathrm{S}^{\prime}$ and $\mathrm{E}^{\prime}$ ), but not of strain and strain rate values. In contrast to our results, one previous study showed significant differences of the peak systolic longitudinal strain between segments with LE $\geq 51 \%$ and LE $1-50 \%{ }^{15}$.

By using the previously proposed cut-off value ${ }^{15}$ for strain of $-6.5 \%$ a sensitivity of $23 \%$ and specificity of $87 \%$ was calculated for the detection of infarcted segments (AUC 0.6, confidence interval $0.53-0.64$ ). A cut-off value of $-10.34 \%$ resulted in a sensitivity of 54 $\%$ and specificity of $59 \%$ (see Table 6).

The intra- and inter-observer variation of our data is congruent with the data of Jarnert et al. and Zeng et al. ${ }^{38-39}$. In general, the variability, which has been assessed in this study, do not differ from previously published data of parametric echocardiography, already clinically integrated and established ${ }^{15}$.

\section{Limitations}

Based on 2D gray scale images the quality of VVI data is strictly dependent on the quality of the echocardiographic loops. A problem of all Strain Rate Imaging algorithms is the huge variability of comparable parameters and values, which are provided by different ultrasound machines and software algorithms. There are neither guidelines, standardized technical settings, nor normal values for each of the different software packages, which complicates the comparison of the different approaches ${ }^{30}$. Although we analyzed the segments following a strict protocol we had to exclude segments in which the tracking failed (feasibility 71\%).

One could also criticize the intra-individual comparison instead of considering a control group. In our opinion, as the infarct is a regional process, there is no better match than the non-infarcted segment of the same patient for an infarcted segment. Otherwise there will always be confounders like age, blood pressure, and heart rate.

\section{Conclusions}

Velocity Vector imaging (VVI) is a clinically feasible approach for strain measurements in infarcted myocardium allowing an accurate assessment of global and regional myocardial function, and a differentiation between infarcted and non-infarcted segments. Further research for the definition of cut-off values and technical standards will be needed to fully integrate this method into clinical practice. The detection of scarred as well as vital, but dysfunctional myocardium by VVI might be useful for multimodal risk stratification and the determination of different therapeutic approaches as well as prognosis in future.

\section{Abbreviations}

AMI: Acute myocardial infarction;

$\mathrm{A}^{\prime}$ : Peak late diastolic tissue velocity $[\mathrm{cm} / \mathrm{s}]$;

$\mathrm{E}^{\prime}$ : Peak early diastolic velocity $[\mathrm{cm} / \mathrm{s}]$;

ECG: Electrocardiography ;

EF: Ejection fraction [\%];

2DS: 2-dimensional peak systolic strain [\%];

2DST: 2-dimensional speckle tracking;

Echo: Echocardiography; 
CAD: Coronary artery disease;

LAD: Left anterior descending artery;

LE: Late Enhancement [\%];

LV: Left ventricle;

MRI: Magnetic resonance Imaging;

PCI: Percutaneous coronary intervention;

RCA: Right coronary artery;

CX: Circumflex artery;

$\mathrm{S}^{\prime}$ : Peak systolic tissue velocity $[\mathrm{cm} / \mathrm{s}]$;

SR: strain rate [s-1];

sSR: peak systolic strain rate [s-1];

SRe: eak early diastolic systolic strain rate [s-1];

ROI: Region of interest;

TDI: Tissue Doppler Imaging;

VVI: Velocity Vector Imaging.

\section{Conflict of Interest}

The authors have declared that no conflict of interest exists.

\section{References}

1. Kloner RA, Przyklenk K. Hibernation and stunning of the myocardium. N Engl J Med 1991; 325(26): 1877-1879.

2. D'Hooge J, Heimdal A, Jamal F, et al. Regional strain and strain rate measurements by cardiac ultrasound: principles, implementation and limitations. Eur J Echocardiogr 2000; 1(3): 154-170.

3. Mirsky I, Parmley WW. Assessment of passive elastic stiffness for isolated heart muscle and the intact heart. Circ Res 1973; 33(2): 233-243.

4. Pavlopoulos H, Nihoyannopoulos P. Strain and strain rate deformation parameters: from tissue Doppler to 2D speckle tracking. Int J Cardiovasc Imaging 2008; 24(5): 479-491.

5. Sutherland GR, Di Salvo G, Claus P, D'Hooge J, Bijnens B. Strain and strain rate imaging: a new clinical approach to quantifying regional myocardial function. J Am Soc Echocardiogr 2004; 17(7): 788-802.

6. Voigt JU, Flachskampf FA. Strain and strain rate. New and clinically relevant echo parameters of regional myocardial function. Z Kardiol 2004; 93(4): 249-258.

7. Weidemann F, Wacker C, Rauch A, et al. Sequential changes of myocardial function during acute myocardial infarction, in the early and chronic phase after coronary intervention described by ultrasonic strain rate imaging. J Am Soc Echocardiogr 2006; 19(7): 839-847.

8. Adamu U, Schmitz F, Becker M, Kelm M, Hoffmann R. Advanced speckle tracking echocardiography allowing a three-myocardial layer-specific analysis of deformation parameters. Eur J Echocardiogr 2009; 10(2): 303-308.

9. Becker M, Hoffmann R, Kuhl HP, et al. Analysis of myocardial deformation based on ultrasonic pixel tracking to determine transmurality in chronic myocardial infarction. Eur Heart J 2006; 27(21): 2560-2566.

10. Becker M, Ocklenburg C, Altiok E, et al. Impact of infarct transmurality on layer-specific impairment of myocardial function: a myocardial deformation imaging study. Eur Heart J 2009; 30(12): 1467-1476.

11. Chan J, Hanekom L, Wong C, Leano R, Cho GY, Marwick TH. Differentiation of subendocardial and transmural infarction using two-dimensional strain rate imaging to assess short-axis and long-axis myocardial function. J Am Coll Cardiol 2006; 48(10): 2026-2033.
12. Gjesdal O, Hopp E, Vartdal T, et al. Global longitudinal strain measured by two-dimensional speckle tracking echocardiography is closely related to myocardial infarct size in chronic ischaemic heart disease. Clin Sci (Lond) 2007; 113(6): 287-296.

13. Leitman M, Lysyansky P, Sidenko $S$, et al. Two-dimensional strain-a novel software for real-time quantitative echocardiographic assessment of myocardial function. J Am Soc Echocardiogr 2004; 17(10): 1021-1029.

14. Chen J, Cao T, Duan Y, Yuan L, Yang Y. Velocity vector imaging in assessing the regional systolic function of patients with post myocardial infarction. Echocardiography 2007; 24(9): 940-945.

15. Jurcut R, Pappas CJ, Masci PG, et al. Detection of regional myocardial dysfunction in patients with acute myocardial infarction using velocity vector imaging. J Am Soc Echocardiogr 2008; 21(8): 879-886.

16. Cheitlin MD, Armstrong WF, Aurigemma GP, et al. ACC/AHA/ASE 2003 Guideline Update for the Clinical Application of Echocardiography: summary article. A report of the American College of Cardiology/American Heart Association Task Force on Practice Guidelines (ACC/AHA/ASE Committee to Update the 1997 Guidelines for the Clinical Application of Echocardiography). J Am Soc Echocardiogrt 2003; 16(10): 1091-1110.

17. Tonti G, Riccardi G, Denaro FM, Trambaiolo P, Salustri A. From digital image processing of colour Doppler M-mode maps to noninvasive evaluation of the left ventricular diastolic function: a dedicated software package. Ultrasound Med Biol 2000; 26(4): 603-611.

18. Lang RM, Bierig M, Devereux RB, et al. Recommendations for chamber quantification: a report from the American Society of Echocardiography's Guidelines and Standards Committee and the Chamber Quantification Writing Group, developed in conjunction with the European Association of Echocardiography, a branch of the European Society of Cardiology. J Am Soc Echocardiogr 2005; 18(12): 1440-1463.

19. Cannesson M, Tanabe M, Suffoletto MS, Schwartzman D, Gorcsan J3rd. Velocity vector imaging to quantify ventricular dyssynchrony and predict response to cardiac resynchronization therapy. Am J Cardiol 2006; 98(7): 949-953.

20. Vannan MA, Pedrizzetti G, Li P, et al. Effect of cardiac resynchronization therapy on longitudinal and circumferential left ventricular mechanics by velocity vector imaging: description and initial clinical application of a novel method using high-frame rate B-mode echocardiographic images. Echocardiography 2005; 22(10): 826-830.

21. Sutherland GR, Hatle L, Claus P, D'Hooge J, Bijnens B. Doppler Myocardial Imaging - A Textbook. Hasselt: BSWK bvba, Scientific Consulting and Publishing; 2006.

22. Maceira AM, Prasad SK, Khan M, Pennell DJ. Normalized left ventricular systolic and diastolic function by steady state free precession cardiovascular magnetic resonance. J Cardiovasc Magn Reson. 2006;8(3):417-426.

23. Butz T, Piper C, Langer C, et al. Diagnostic superiority of a combined assessment of the systolic and early diastolic mitral annular velocities by tissue Doppler imaging for the differentiation of restrictive cardiomyopathy from constrictive pericarditis. Clin Res Cardiol 2010; 99(4): 207-15

24. Bach DS, Armstrong WF, Donovan CL, Muller DW. Quantitative Doppler tissue imaging for assessment of regional myocardial velocities during transient ischemia and reperfusion. Am Heart J. 1996; 132(4): 721-725.

25. Edvardsen T, Aakhus S, Endresen K, Bjomerheim R, Smiseth $\mathrm{OA}$, Ihlen $\mathrm{H}$. Acute regional myocardial ischemia identified by 2-dimensional multiregion tissue Doppler imaging technique. J Am Soc Echocardiog 2000; 13(11): 986-994. 
26. Heimdal A, Stoylen A, Torp H, Skjaerpe T. Real-time strain rate imaging of the left ventricle by ultrasound. J Am Soc Echocar$\operatorname{diog} 1998 ; 11(11):$ 1013-1019.

27. Rademakers F, Van de Werf F, Mortelmans L, Marchal G, Bogaert J. Evolution of regional performance after an acute anterior myocardial infarction in humans using magnetic resonance tagging. J Physiol 2003; 546(Pt 3): 777-787.

28. Van Leuven SL, Waldman LK, McCulloch AD, Covell JW. Gradients of epicardial strain across the perfusion boundary during acute myocardial ischemia. Am J Physiol 1994; 267(6 Pt 2): 2348-2362.

29. Masuda K. Assessment of Dyssynchronous Wall Motion During Acute Myocardial Ischemia Using Velocity Vector Imaging. JACC Cardiovasc Imaging 2008; 1(2): 210-20.

30. Amundsen BH, Helle-Valle T, Edvardsen T, et al. Noninvasive myocardial strain measurement by speckle tracking echocardiography: validation against sonomicrometry and tagged magnetic resonance imaging. J Am Coll Cardiol 2006; 47(4): 789-793.

31. Becker M, Bilke E, Kuhl H, et al. Analysis of myocardial deformation based on pixel tracking in two dimensional echocardiographic images enables quantitative assessment of regional left ventricular function. Heart 2006; 92(8): 1102-1108.

32. Becker M, Lenzen A, Ocklenburg C, et al. Myocardial deformation imaging based on ultrasonic pixel tracking to identify reversible myocardial dysfunction. J Am Coll Cardiol 2008; 51(15): 1473-1481.

33. Cho GY, Chan J, Leano R, Strudwick M, Marwick TH. Comparison of two-dimensional speckle and tissue velocity based strain and validation with harmonic phase magnetic resonance imaging. Am J Cardiol 2006; 97(11): 1661-1666.

34. Voigt JU. Tissue doppler for the assessment of LV function what makes sense? Z Kardiol 2005; 94 (Suppl 4): 38-42.

35. Marwick TH. Measurement of strain and strain rate by echocardiography: ready for prime time? J Am Coll Cardiol 2006; 47(7): 1313-1327.

36. Butz T, van Buuren F, Mellwig KP, Langer C, Plehn G, Meissner A, Trappe HJ, Horstkotte D, Faber L. Two-dimensional strain analysis of the global and regional myocardial function for the differentiation of pathologic and physiologic left ventricular hypertrophy: A study in athletes and in patients with hypertrophic cardiomyopathy. International Journal of Cardiovascular Imaging 2010; in press

37. Butz T, van Buuren F, Mellwig K, et al. Systolic and early diastolic left ventricular velocities assessed by tissue Doppler imaging in 100 top-level handball players. European Journal of Cardiovascular Prevention \& Rehabilitation 2010; 17: 342-348

38. Jarnert C, Melcher A, Caidahl K, Persson H, Ryden L, Eriksson MJ. Left atrial velocity vector imaging for the detection and quantification of left ventricular diastolic function in type 2 diabetes. Eur J Heart Fail 2008; 10(11): 1080-1087.

39. Zeng S, Zhou QC, Peng QH, et al. Assessment of regional myocardial function in patients with dilated cardiomyopathy by velocity vector imaging. Echocardiography 2009; 26(2):163-170. 